\title{
Migrantische Ökonomien zwischen Potentialorientierung und Differenzmarkierung. Konzeption und Erträge eines „,prä-postmigrantischen“" Forschungsgegenstands
}

\author{
Charlotte Räuchle $^{1}$ and Henning Nuissl ${ }^{2}$ \\ ${ }^{1}$ Institut für Geographie/Institut für Migrationsforschung und Interkulturelle Studien (IMIS), \\ Universität Osnabrück, Osnabrück, Germany \\ ${ }^{2}$ Geographisches Institut, Humboldt-Universität Berlin, Berlin, Germany \\ Correspondence: Charlotte Räuchle (charlotte.raeuchle@uni-osnabrueck.de)
}

Received: 26 June 2018 - Revised: 27 October 2018 - Accepted: 12 November 2018 - Published: 15 January 2019

Kurzfassung. In public discourse, issues of migration and immigration were for a long time perceived "only" as an integration problem. Nevertheless, a potential-oriented view of migration has been postulated recently and also implemented in practice. Thus, especially in large cities, various political programmes have been adopted that aim at commodifying the potential of migrant economies for urban development. This potential-oriented perspective is, however, decidedly "pre-post-migrant", since it requires some kind of distinctive concept of the "migrant" in general and migrant economies in particular. Based on a project examining the importance of migrant economies for urban development in smaller cities, this article discusses the topic as an issue of research and local politics in a post-migrant perspective. Finally, uncertainties on how to deal with migrant economies also arise from the field itself and various challenges remain for a post-migrant "production of urban space".

\section{Einleitung}

Migrantische Ökonomien sind inzwischen ein etabliertes Forschungsthema in der Geographie. Sie werden aus unterschiedlichen Blickwinkeln untersucht, nicht zuletzt im Hinblick auf das ihnen innewohnende Potential für die städtische und regionale Entwicklung (z.B. Aver, 2013; FischerKrapohl und Waltz, 2005; Fürst und Balke, 2013; Kayser et al., 2008; Nuissl und Schmiz, 2015; Schaland, 2012; Schuleri-Hartje et al., 2005). Insbesondere in Großstädten wird vielfach versucht, dieses Potential im Rahmen von Stadtentwicklungsstrategien, Branchenkonzepten, Quartiersmanagements oder Place-Branding-Aktivitäten auch praktisch in Wert zu setzen und zu fördern (z.B. Aytar und Rath, 2012; Pütz und Rodatz, 2013; Schmiz, 2017). Im Kontext eines ,postmigrantischen Paradigmas“, das im Kern zur Auflösung einer differenzmarkierenden und homogenisierenden Betrachtung von Migrationsphänomenen auffordert und Migrations- als Gesellschaftsforschung konzipiert, erscheint dieser potentialorientierte Zugriff auf migrantische Ökonomien dabei zunächst dezidiert ,prä-postmigrantisch“:
Denn er benötigt einen wie auch immer gearteten distinktiven Begriff des „Migrantischen“ im Allgemeinen und der migrantischen Ökonomien im Besonderen. Dieser impliziert wiederum zwangsläufig sowohl eine Differenz zum „NichtMigrantischen“ als auch eine Homogenisierung des „Migrantischen" selbst.

Der vorliegende Beitrag geht der Frage nach, inwieweit eine postmigrantische Perspektive auf migrantische Ökonomien überhaupt möglich ist und welche Anforderungen sich aus einer solchen Perspektive für die politische und planerische Handhabung urbaner Räume ergeben können. Hierzu werden der Ansatz und die Ergebnisse eines DFG-Projekts, das die Potentiale migrantischer Ökonomien für die Stadtund Regionalentwicklung kleinerer Großstadtregionen untersucht, reflektiert und zu zentralen Argumenten des „Postmigrantischen“ in Beziehung gesetzt. Im Folgenden wird zunächst das Forschungsfeld der migrantischen Ökonomien eingeführt (2), um es anschließend aus einer postmigrantischen Perspektive zu beleuchten (3). Sodann wird am Beispiel der eigenen Forschungsergebnisse reflektiert, ob bzw. 
wie die Umsetzung des ,postmigrantischen Paradigmas“ forschungspraktisch gelingen kann (4). Hierauf folgt eine Diskussion der ,theoretischen“ Sinnhaftigkeit des Forschungsfelds der migrantischen Ökonomien sowie eine erste Auslotung der ,praktischen“ Möglichkeit der Etablierung einer postmigrantischen Stadtentwicklungspolitik (5). Ein kurzes Resümee (6) beschließt den Beitrag.

\section{Migrantische Ökonomien als Potential für die Stadtentwicklung}

Der Beitrag migrantischer Ökonomien zur gesellschaftlichen Wohlfahrt ist in der Literatur vielfach belegt. Das gilt insbesondere für die angelsächsische Forschung, die sich dem Thema bereits seit den 1970er Jahren widmete (z.B. Light, 1972; Portes et al., 2002; Waldinger et al., 1990). Mit ähnlichen Ergebnissen werden migrantische Ökonomien seit den 1990er Jahren verstärkt auch im deutschsprachigen Kontext erforscht (z.B. Kayser et al., 2008; Schmiz, 2011; Stock, 2013; Yildiz und Mattausch, 2009).

In Bezug auf die Gesamtwirtschaft stellen Leicht und Langhauser (2014: 6f.) fest, dass sich die Zahl selbstständiger Migrant*innen in Deutschland seit den 1990er Jahren annähernd verdreifacht hat und mittlerweile eine dreiviertel Million beträgt. Mit 2,2 Millionen Beschäftigten stellen migrantische Ökonomien - verstanden als Gesamtheit aller Unternehmen, die von Personen mit Migrationshintergrund geführt werden $-18 \%$ aller Arbeitsplätze in inhabergeführten mittelständischen Unternehmen. Vor diesem Hintergrund wird insbesondere in praxisorientierten Studien regelmäßig darauf hingewiesen, dass diese Unternehmen in erheblichem Maße zur volkswirtschaftlichen Gesamtleistung sowie generell zum Wirtschaftsleben in Deutschland beitragen (z.B. Bertelsmann Stiftung, 2016).

Auf der lokalen Ebene verbinden sich mit migrantischen Ökonomien ebenfalls Potentiale, die allerdings in der deutschsprachigen Debatte im Vergleich zur angelsächsischen noch wenig thematisiert werden. Gleichwohl werden seit einigen Jahren verstärkt die konkreten Beiträge migrantischer Ökonomien zur Profilierung und zum wirtschaftlichen Erfolg von Städten und Regionen herausgestellt (z.B. Floeting, 2009; Schuleri-Hartje et al., 2005; Yildiz, 2011). Dabei lassen sich fünf Funktionen voneinander unterscheiden, die migrantische Ökonomien für die Regional-, Stadt- und Quartiersentwicklung prinzipiell übernehmen können (Nuissl und Schmiz, 2015), wobei die beiden letztgenannten explizit die lokale Ebene betreffen:

1. die beschäftigungspolitische Funktion, die auch auf der stetig wachsenden Ausbildungsbereitschaft der Betriebe der migrantischen Ökonomien beruht (Leicht und Langhauser, 2014: 62ff.) und die vielen Personen einen Zugang zum ersten Arbeitsmarkt eröffnet (Mestres, 2010: 38f.; Rath und Eurofound, 2011: 1f.);
2. die gesellschaftspolitische Funktion, die den Beitrag migrantischer Ökonomien zur Sicherstellung einer umfassenden gesellschaftlichen Teilhabe von Immigrant*innen betrifft und nicht zuletzt von erfolgreichen Unternehmer*innen mit Migrationshintergrund symbolisiert wird (Unabhängige Kommission „Zuwanderung“", 2001: 225f.);

3. die ökonomische Funktion, die sich aus der Bedeutung migrantischer Ökonomien für die Herausbildung von Wertschöpfungsketten ergibt und unter anderem auf deren Innovationspotential zurückgeht (Hillmann, 2011);

4. die quartiersbelebende Funktion, die sich in vielen urbanen Teilräumen beobachten lässt, in denen es häufig kleine Dienstleistungsunternehmen der migrantischen Ökonomien sind, die sowohl die Nahversorgung sichern als auch eine gewisse nachbarschaftliche Kohäsion aufrechterhalten (Idik und Schnetger, 2004; Rath und Eurofound, 2011);

5. die imagebildende Funktion, die auf der ethnokulturellen Vermarktbarkeit von migrantischen Ökonomien als Bestandteil von Imagebildungs- und Marketingstrategien von Städten beruht (Aytar und Rath, 2012; Shaw, 2011).

Vor allem im angelsächsischen Raum werden migrantische Ökonomien nicht nur als stadtentwicklungspolitisches Potential wahrgenommen, sondern als solches auch proaktiv gefördert. Im kanadischen Toronto beispielsweise ist es mit Hilfe des Stadtentwicklungsinstruments der Business Improvement Areas gelungen, migrantisch geprägte Viertel als touristische Destinationen und weiche Standortfaktoren für die Ansiedlung der Wissensökonomie zu vermarkten (Ipsen et al., 2005; Luk und Phan, 2005; Zhuang, 2008). Eine ähnlich erfolgreiche Vermarktung von Quartieren als „ethnisch“ ist auch für viele andere Städte dokumentiert (z.B. Aytar und Rath, 2012; Shaw, 2011; van der Horst und Ouwehand, 2012), wobei die Ambivalenz der entsprechenden Strategien zwischen einem der kapitalistischen Verwertungslogik unterworfenen city branding einerseits und einer auf Chancengleichheit und Teilhabe zielenden Unterstützung migrantischer Unternehmer*innen deutlich herausgearbeitet wurde (z.B. Dinnie, 2011; Donald et al., 2009; Kavaratzis und Ashworth, 2005). Auch in einigen deutschen Städten lassen sich derartige Inwertsetzungsstrategien beobachten (z.B. FischerKrapohl, 2011 für Dortmund; Henn und Behling, 2010 für Leipzig; Schmiz, 2017 für Berlin). Insgesamt kann aber festgehalten werden, dass in der deutschsprachigen Literatur die Potentiale migrantischer Ökonomien überwiegend mit Blick auf großskalige Zusammenhänge diskutiert werden und, sofern überhaupt die lokale Ebene thematisiert wird, fast ausschließlich Großstädte in den Blick geraten. 


\section{Migrantische Ökonomien in postmigrantischer Perspektive}

Es dürfte bereits deutlich geworden sein, dass das „Migrantische" in einer potentialorientierten Sicht auf migrantische Ökonomien typischerweise im Gegensatz zu einem wie auch immer definierten „Nicht-Migrantischen“ gedacht wird. Darüber hinaus wird vielfach zumindest implizit davon ausgegangen, dass sich gleichsam alle migrantischen Ökonomien (im Vergleich zu nicht-migrantischen) durch charakteristische Stärken auszeichnen. Das Konzept des Postmigrantischen fordert demgegenüber dazu auf, die Engführungen einer sowohl separierenden als auch homogenisierenden Perspektive auf Migrationsphänomene allgemein aufzubrechen.

Auch wenn verschiedene Spielarten des Postmigrantischen existieren, lassen sich vier zentrale Kritikpunkte an der „traditionellen“ Art und Weise der Thematisierung von Migrationsphänomenen herausarbeiten, die allen in der Literatur vorfindlichen postmigrantischen Ansätzen gemein sind (Mecheril, 2014: 108ff.): Kritisiert werden

- das „nationalstaatliche Integrationsdispositiv“,

- die „Defizitperspektive auf Migration“,

- die „Reduktion migrationsgesellschaftlicher Wirklichkeit auf (klassische) Einwanderung" sowie

- die „Repräsentationsverhältnisse (Wer spricht über wen?)“.

Im Zentrum postmigrantischer Ansätze steht das Petitum, „den engen Kreis der Migrationsmarkierten [...] zugunsten der Konzeption einer postmigrantischen Gesellschaft, die alle zu ,Betroffenen' der Migration und zu GestalterInnen der dadurch konstituierten Verhältnisse erklärt", zu überschreiten (Bojadzijev und Römhild, 2014: 18f.). Damit zielen diese Ansätze auf die Überwindung etablierter sozialer und sprachlicher Praxen der Differenzierung und Grenzziehung entlang des Phänomens der (internationalen) Migration (Foroutan, 2016: 248) sowie die Aufdeckung der von diesen Praxen hervorgebrachten Verhältnisse sozialer Ungleichheit und Hierarchie. Konsequent weitergedacht, impliziert diese Positionierung, dass zwischen Migrations- und Gesellschaftsforschung gar nicht sinnvoll unterschieden werden kann, da Migration stets ein gesamtgesellschaftlich relevantes und auch konstruiertes - Phänomen ist. Demnach bedarf es keiner eigenständigen Migrationsforschung, sondern es gilt, Gesellschaftsforschung zu „migrantisieren“ (Bojadzijev und Römhild, 2014: 20): „Wir konzipieren die postmigrantische Gesellschaft als gesellschaftstheoretische Perspektivierung, mit der die empirische Tatsache ins Zentrum gerückt wird, dass Migration nicht als Ausnahme von nationalen Vergesellschaftungsprozessen begriffen werden kann, sondern zentrale Normalität von Gesellschaft ist - gleichwohl einer Gesellschaft in der die institutionelle und allägliche Reprodukti- on von Rassismus nicht einfach verschwindet, sondern neue Formen und Wege nimmt“" (Espahangizi et al., 2016: 15).

Trotz ihrer Nähe zu traditionellen Ansätzen der Migrationsforschung lassen sich in den gegenwärtigen wissenschaftlichen Debatten zu migrantischen Ökonomien auch postmigrantische Argumente und Ansatzpunkte finden. Insbesondere drei Aspekte können dabei hervorgehoben werden: die Hinterfragung des Migrations- und Ethnizitätsbegriffs, die Berücksichtigung von Transnationalität sowie eine kritische Distanz zur „neoliberalen Inwertsetzung“ von Migration und Migrantischem:

1. Studien zu migrantischen Ökonomien beginnen häufig mit einer Diskussion der verwendeten Begrifflichkeiten. Dabei werden nicht nur gleichsam statistische Begriffe der „Besonderung“ des „Migrantischen“ wie „Ausländer*in“, „Migrant*in“ oder „Migrationshintergrund" kritisch reflektiert, sondern es wird zunehmend auch infrage gestellt, ob Ethnizität ,als Kerncharakteristikum der Selbstständigkeit von Migranten/Migrantinnen“ ausgewiesen werden sollte (Schmitt, 2015: 21; Glick Schiller und Çağlar, 2011; Pécoud, 2010). Denn die Ethnisierung unternehmerischen Handelns läuft Gefahr, dieses Handeln ausschließlich als Ausdruck einer bestimmten Gruppenzugehörigkeit zu interpretieren und dessen Einbettung in heterogene Netzwerke und strukturelle Rahmenbedingungen zu negieren (Schmitt, 2015: 21). Des Weiteren werden meist nur bestimmte Unternehmer*innen ,ethnisch“ markiert: Beispielsweise Koreaner*innen in Los Angeles, Chines*innen in New York, Nordafrikaner*innen in Lyon oder Türk*innen in Berlin, die kleine Geschäfte betreiben (Timm, 2000: 364).

2. Insbesondere soweit konkrete Akteure bzw. Unternehmer*innenpersönlichkeiten in den Blick genommen werden, löst sich die Forschung zu migrantischen Ökonomien typischerweise von nationalstaatlich grundierten Identitätskategorien, betont stattdessen die Bedeutung hybrider Identitätskonstruktionen und beruft sich auf das Konzept des Transnationalismus (z.B. Schmiz, 2011; Yildiz, 2016). Einem genuin postmigrantischen Forschungsparadigma folgt sie in dieser Hinsicht aber nur teilweise. Denn dieses fordert nicht allein eine akteurszentrierte, sondern auch eine strukturelle Thematisierung und Sichtbarmachung von transnationalen Vernetzungen und Lebenswelten (Foroutan, 2016).

3. Zunächst ist zu konstatieren, dass eine potentialorientierte Perspektive auf Migration, mit der die Forschung zu migrantischen Ökonomien eng verbunden ist, (auch) aus postmigrantischer Perspektive einen anderen Blick auf Migrationsphänome wirft als eine vormals vorherrschende problemorientierte Thematisierung (vgl. Häußermann und Kapphan, 2008). Vor allem aber erfolgt im Kontext der Auseinandersetzung 
mit migrantischen Ökonomien mittlerweile auch eine dezidiert kritische Reflexion der Interessen, Konflikte und Allianzen, die im Zuge der Inwertsetzung migrantischer Ökonomien für die Stadt- und Regionalentwicklung entstehen (z.B. Schmiz, 2017). In diesem Zusammenhang wurden nicht nur das Leitbild des ,aktivierenden Staats“ und die Prämisse des „Förderns und Forderns" dekonstruiert (Ülker, 2016). Zugleich wurden die Potential-, Querschnitts-, Netzwerk- und Sozialraumorientierung kommunaler Integrationsstrategien (Pütz und Rodatz, 2013: 167) als Ausdruck einer Stadtpolitik entlarvt, die auch die migrantischen Ökonomien am Ort der kapitalistischen Verwertungslogik der „unternehmerischen Stadt“ unterwirft (vgl. Harvey, 1989; Rodatz, 2014). Die neoliberale Grundierung der Potentialorientierung in der Thematisierung von Migration im Allgemeinen und der Erforschung von migrantischen Ökonomien im Besonderen wird in der neueren Literatur zu migrantischen Ökonomien demnach umfassend rezipiert und reflektiert (z.B. Husseini de Araújo und Weber, 2014).

Die mit dem Konzept des Postmigrantischen vorgezeichnete kritische Perspektive auf jede Art von Migrationsforschung, die ihren Forschungsgegenstand über das ,Migrantische“ definiert (statt ihn zu dekonstruieren), ist also - zumindest in Teilen - auch in der wissenschaftlichen Auseinandersetzung mit migrantischen Ökonomien bereits explizit aufgegriffen worden. Es fehlt jedoch an konkreten Versuchen auszuloten, inwiefern sich diese Perspektive mit der anwendungsorientierten Untersuchung migrantischer Ökonomien und ihres Beitrags zur Stadt- und Regionalentwicklung versöhnen lässt bzw. bis zu welchem Punkt ein solches Erkenntnisinteresse auch aus postmigrantischer Perspektive legitim und zielführend sein könnte.

\section{Migrantische Ökonomien in der Forschungspraxis}

Vor diesem Hintergrund wird im Folgenden der Versuch unternommen, Ansätze und Ergebnisse eines eigenen Forschungsprojekts aus postmigrantischer Perspektive zu reinterpretieren. Das Konzept des Postmigrantischen dient hier also nicht als (heuristischer) Analysebegriff, sondern als Interpretationsfolie zur Einordung einer bereits vorliegenden Untersuchung. Anhand zweier Fallstudien untersuchte das Projekt migrantische Ökonomien als lokalpolitisches Handlungsfeld. Die forschungsleitende Frage war, ob und, wenn ja, wie migrantische Ökonomien als Potential für die Stadtund Regionalentwicklung wahrgenommen werden und welche Rahmenbedingungen für ihre Entwicklung und Entfaltung in den beiden untersuchten Fällen existieren. Als Fallstudienregionen wurden zwei außerhalb der großen Agglomerationen gelegene Stadt-Umland-Räume gewählt und damit ein - zuweilen als Regiopole (Aring und Reuther, 2008) ausgewiesener - Raumtyp, der in der Debatte um migranti- sche Ökonomien bislang weitgehend vernachlässigt wurde: die IHK-Regionen Braunschweig und Rostock. Der hauptsächliche Forschungsfokus lag dabei auf den beiden gleichnamigen kleineren Großstädten. Das Projekt kombinierte quantitative und qualitative Methoden der empirischen Sozialforschung: statistische Sekundär- und Dokumentenanalysen, besonders aber Expert*innen- und problemzentrierte Interviews, Gruppendiskussionen, teilnehmende Beobachtungen und Feldbegehungen. Interviewtranskripte und Beobachtungsprotokolle wurden mithilfe einer qualitativen Inhaltsanalyse und einer Deutungsmusteranalyse interpretiert. Die empirischen Erhebungen fanden zwischen April 2014 und November 2015 statt. Den jeweiligen methodischen Ansätzen lagen dabei unterschiedliche Erkenntnisinteressen und definitorische Zugriffe auf migrantische Ökonomien zugrunde, wie Tabelle 1 verdeutlicht.

In der empirischen Vorgehensweise wurde insofern der Versuch unternommen, (auch) die postmigrantische Perspektive aufzugreifen, als der zentrale Forschungsgegenstand, die migrantischen Ökonomien in den beiden Fallstudienregionen, vorab nur im Sinne einer Arbeitsdefinition abgegrenzt wurde, die dann im Zuge der Feldforschung um die Sichtweise(n) der befragten Akteure angereichert wurde. In den folgenden Unterabschnitten werden zunächst die im Forschungsprojekt erzielten Teilergebnisse dargestellt sowie die Auswahl der definitorischen Zugriffe gerechtfertigt, um diese Ergebnisse sowie das ihnen zugrunde liegende Verständnis migrantischer Ökonomien anschließend aus postmigrantischer Perspektive zu reflektieren.

\section{1 Ökonomischer Kontext: Bestand und Verortung migrantischer Ökonomien}

Die Erhebung des Bestands migrantischer Ökonomien in den beiden Fallstudienregionen diente der Einschätzung ihrer Größe, ihrer sektoralen und räumlichen Struktur sowie der Identifikation der ökonomisch besonders bedeutsamen Zuwanderungsmilieus. Von den im Rahmen des Projekts interviewten Vertreter*innen der kommunalpolitischen Praxis wurde immer wieder betont, dass „exakte Daten“ die Voraussetzung dafür seien, Förderprogramme für migrantische Unternehmer*innen zu entwickeln und Gelder zu beantragen. Die Ermittlung quantitativer Daten und Fakten zu migrantischen Ökonomien ,vor Ort“ spielte daher mit Blick auf den Anwendungsbezug und die Einbindung von Praxispartner*innen ins Projekt eine wichtige Rolle.

Allerdings unterliegt gerade die statische Erhebung migrantischer Ökonomien einer Reihe methodischer Einschränkungen (Hillmann und Sommer, 2011). So besteht in einer sekundärstatistischen Auswertung keine Möglichkeit, hinter die den verwendeten Datensätzen zugrundeliegenden Kategorien zurückzugehen. Im Fall einer Studie zu migrantischen Ökonomien auf lokaler Ebene bedeutet dies, dass die Definition von migrantischen Ökonomien als die Summe der selbstständigen Erwerbstätigkeiten von Personen nicht- 
Tabelle 1. Untersuchte Faktoren, angewendete Methoden und Definitionen.

\begin{tabular}{|c|c|c|}
\hline Faktoren & Methoden & Definition „migr. Ökonomien“ \\
\hline $\begin{array}{l}\text { (1) ökonomischer Kontext: } \\
\text { Umfang und Struktur der mi- } \\
\text { grantischen Ökonomien ,vor } \\
\text { Ort"“ }\end{array}$ & Statistische Sekundäranalyse & $\begin{array}{l}\text {... selbstständige Erwerbstätigkeit von Perso- } \\
\text { nen nicht-deutscher Staatsangehörigkeit (nach } \\
\text { Rath und Swagerman, 2016: 153) }\end{array}$ \\
\hline $\begin{array}{l}\text { (2) institutioneller Kontext: } \\
\text { Akteure, deren Konstellatio- } \\
\text { nen und politische Program- } \\
\text { me/Maßnahmen }\end{array}$ & Dokumentenanalyse, Interview & $\begin{array}{l}\text {... selbstständige Erwerbstätigkeit von Perso- } \\
\text { nen mit Migrationshintergrund (einschließlich } \\
\text { deren Nachfolgegeneration) sowie abhängige } \\
\text { Beschäftigung in Betrieben, die von solchen } \\
\text { Personen geführt werden (nach Schuleri-Hartje } \\
\text { et al., 2005: 21) }\end{array}$ \\
\hline $\begin{array}{l}\text { (3) sozio-kultureller Kontext: } \\
\text { Wahrnehmungen der migranti- } \\
\text { schen Ökonomien }\end{array}$ & Interview, Gruppendiskussion & $\begin{array}{l}\text {.. das, was bestimmte Menschen bzw. Perso- } \\
\text { nengruppen darunter verstehen }\end{array}$ \\
\hline
\end{tabular}

deutscher Staatsangehörigkeit gleichsam alternativlos ist. Denn für die lokale Gewerbestatistik wird regelmäßig nur die Staatsangehörigkeit von Unternehmer*innen erhoben. Ohnehin ist allerdings auch der „Alternativbegriff“ des Migrationshintergrunds aufgrund seines latent von der Mehrheitsgesellschaft ausgrenzenden Charakters und seiner gleichzeitigen inhaltlichen Unbestimmtheit nicht unproblematisch (Nieswand und Drotbohm, 2014).

Die Ergebnisse der sekundärstatistischen Analyse illustrieren gleichsam idealtypisch, auf welche Art von Befunden eine quantitative Untersuchung migrantischen Unternehmertums abzielt. So ließ sich feststellen, dass in Braunschweig von 17309 registrierten Betrieben 1945 bzw. 11,2\% von Personen mit ausländischer Staatsangehörigkeit geführt wurden (Stichtag: 30 Juni 2014). Die größte Gruppe unter ihnen stellten dabei Unternehmer*innen mit polnischer (691 bzw. 4\%), gefolgt von solchen mit türkischer (328 bzw. 1,9\%) und italienischer (118 bzw. 0,7\%) Staatsangehörigkeit (Stadt Braunschweig, Abteilung Ordnungs- und Gewerbeangelegenheiten; eigene Berechnungen). Im Vergleich dazu wurden in Rostock von 14400 registrierten Betrieben nur 808 bzw. $6 \%$ von Personen mit ausländischer Staatsangehörigkeit geführt (Stichtag: 30 Juni 2014). $128(0,9 \%)$ dieser Unternehmen gehörten vietnamesischen, $71(0,5 \%)$ litauischen und $51(0,4 \%)$ ukrainischen Staatsangehörigen (Hansestadt Rostock, Abteilung Gewerbeangelegenheiten; eigene Berechnungen). In beiden Städten waren die meisten der den migrantischen Ökonomien zugeordneten Unternehmen im Wirtschaftszweig „Sonstige“ angesiedelt, gefolgt von „Handwerk“ und „Handel“.

Im Hinblick auf die räumliche Struktur ließ sich weder in Braunschweig noch in Rostock eine räumliche Konzentration migrantischer Ökonomien feststellen. Zwar gab es in Braunschweig kleinere Ansammlungen von migrantisch geführten Betrieben; diese waren jedoch - auch nach Ansicht der vor Ort interviewten Personen - nicht prägend für ihre unmittelbare Nachbarschaft, geschweige denn, dass sie über diese Nachbarschaft hinaus ausgestrahlt hätten.

Die Erhebung des quantitativen Umfangs sowie die Lokalisierung migrantischer Ökonomien liegen im Interesse von (kommunaler) Politik und Verwaltung und sind zum Beispiel für Quartiersentwicklungs- und Branding-Strategien relevant. Die scheinbare Eindeutigkeit quantitativer Fakten und die Bezugnahme auf klar abgegrenzte (administrative) Raumeinheiten lassen sich in stadtentwicklungspolitische Strategien und Handlungsanweisungen übersetzen. Aus postmigrantischer Perspektive birgt diese Herangehensweise jedoch mindestens zwei konzeptionelle Fallen:

1. Die statistische Reduktion des Begriffs der migrantischen Ökonomien auf die selbstständige Erwerbstätigkeit von Personen nicht-deutscher Staatsangehörigkeit zeigt beispielhaft, wie wenig gegenstandsangemessen eine schematische Differenzierung sozialer Phänomene entlang der dichotomen Differenzkategorien migrantisch und nicht-migrantisch häufig ist: Eine Teilgruppe von Unternehmer*innen wird allein aufgrund ihrer Staatsangehörigkeit „besondert“, ohne dass erkennbar wäre, welche Relevanz dieses demographische Merkmal für ihr ökonomisches Handeln und ihr Unternehmertum hat.

2. Zählung und „Mapping“ migrant*innengeführter Unternehmen „,verräumlichen“ das Phänomen der migrantischen Ökonomien, indem dessen Vorkommen in einem vorab definierten Raumausschnitt erhoben wird (Glasze und Pott, 2014). Dies steht nicht nur im Widerspruch zu neueren Ansätzen in der Sozial-, Kultur- und Wirtschaftsgeographie, die - unabhängig von ihrer jeweiligen theoretischen Spielart - die soziale Konstruktion von räumlichen Phänomenen hervorheben, der eine gleichsam „vor“ der geographischen Forschung vorgenommene Definition von (leeren) Räumen (bzw. Con- 
tainern) nicht gerecht werden kann. Auch aus postmigrantischer Sicht ist dieses Vorgehen unbefriedigend, da die vorgängigen Grenzziehungen im Raum ,das theoretisch Interessante“ an den migrantischen Ökonomien - z.B. ihre Prägung durch Migrationserfahrungen, ihre transnationalen Bezüge und die mutmaßlich hybriden Identitäten ihrer Protagonist*innen - gleichsam abschneiden.

Die beiden skizzierten Kritikpunkte wurden auf der einen Seite zwar nicht erst aus einer postmigrantischen Perspektive heraus, sondern bereits seit Längerem vorgebracht. Nichtsdestotrotz werden sie vor dem Hintergrund neuerer postmigrantischer Debatten um das Verhältnis von Stadt und Migration aber verstärkt relevant, zieht und reproduziert doch die statistische Analyse von migrantischen Ökonomien eine Grenze sowohl zwischen dem ,Wir“ und den „Anderen“ als auch zwischen geographischen (Teil-) Räumen, die der Lebensrealität einer zunehmenden Zahl von Stadtbürger*innen und zwar nicht nur solcher mit Migrationserfahrung - kaum noch entspricht. Auf der anderen Seite ließe sich allerdings zugunsten einer moderat postmigrantisch informierten Erforschung migrantischer Ökonomien, die an einem (wie auch immer) definierten Begriff des „Migrantischen“ festhält und auch Sekundäranalysen amtlicher Statistiken einbezieht, einwenden, dass der gänzliche Verzicht auf Differenzmarkierungen mit einem Verlust an Orientierungs- und Thematisierungsmöglichkeiten einhergeht - denn es ist eine linguistische, psychologische und auch soziologische Binsenweisheit, dass eine Identität (nicht zuletzt von Forschungsgegenständen) nur über Differenzmarkierungen herstellbar ist.

\subsection{Institutioneller Kontext: Governance migrantischer Ökonomien}

Die im vorangegangenen Abschnitt behandelte raumbezogene Bestandsaufnahme migrantischer Ökonomien flankierte im Rahmen der Projektarbeit eine Governance- und Politikfeldanalyse. Letztere zielte darauf $\mathrm{ab}$, den institutionellen Kontext zu erfassen, in dem sich in Braunschweig und Rostock migrantische Ökonomien entfalten, sowie die einzelnen Akteure und deren Aktivitäten (Programme und Maßnahmen) zu identifizieren, die diesen Kontext und damit die Handlungsspielräume migrantischer Unternehmer*innen definieren und die nicht zuletzt dafür maßgeblich sind, inwieweit deren Interessen in lokalen Politiken aufgegriffen werden (Kloosterman and Rath, 2011; Ram et al., 2012; Rath und Swagerman, 2016). Da einerseits der Zugriff auf Migrant*innen über den statistisch definierten Migrationshintergrund vielen lokalpolitischen Programmen zugrunde liegt sowie andererseits die Logik des GovernanceAnsatzes, basierend auf der Bildung spezifischer Akteursgruppen politische Strukturen zu analysieren, die Zusammenfassung migrantischer Ökonomien zu einer Gruppe erforderlich macht, wurde hier auf die Definition von Schuleri-
Hartje et al. (2005) zurückgegriffen. Im Ergebnis konnte erstens eine Einschätzung der Relevanz migrantischer Ökonomien als lokalpolitisches Handlungsfeld gewonnen werden. Zweitens wurde herausgearbeitet, welche Faktoren die Entwicklung migrantischer Ökonomien ,,vor Ort“ befördern oder behindern können.

Akteure: Sowohl in Braunschweig als auch in Rostock konnte eine Vielzahl von Akteuren identifiziert werden, die für die kommunalpolitische Wahrnehmung und Adressierung von migrantischen Ökonomien relevant sind: einzelne migrantische Unternehmer*innen, Migrant*innenselbstorganisationen, Handels- und Handwerkskammern, lokale Wirtschafts- und Start-up-Netzwerke, Wirtschaftsförderungsreferate sowie Abteilungen für Integration und Soziales in den Stadtverwaltungen, Arbeitsämter und private Bildungseinrichtungen - um nur die Wichtigsten zu nennen (Räuchle, 2016). Als besonders einflussreich haben sich Akteure aus Wirtschaft und Zivilgesellschaft erwiesen. Der öffentliche Sektor spielt meist nur insoweit eine Rolle, als Fragen der Sozial- und Integrationspolitik auch migrant*innengeführte Unternehmen betreffen. Eine organisierte Interessenvertretung migrantischer Unternehmer*innen, etwa durch ,ethnisch“ definierte Unternehmer*innenverbände, gibt es nur einmal in Braunschweig.

Beziehungen und Netzwerke: Migrantische Unternehmer*innen sind in Braunschweig und Rostock kaum in bestehende Governance-Strukturen integriert. Nur eine Minderheit von ihnen verfügt über Kontakte zu lokalen Initiativen und Netzwerken, in denen kommunalpolitisch (und auch betriebswirtschaftlich) relevante Informationen ausgetauscht und Entscheidungen vorbereitet werden. Ob diese wenigen Unternehmer*innen in der Lage sind, die Interessen der höchst diversen migrantischen Ökonomien insgesamt zu vertreten, ist fraglich. Diese Ergebnisse entsprechen denen anderer Studien, die auf lokaler Ebene ebenfalls nur spärliche Kontakte zwischen politischen Akteuren und migrantischen Unternehmer*innen vorgefunden haben (Schmiz, 2011). Zudem verweist die Frage der Repräsentanz unternehmerischer Interessen in der Lokalpolitik auf die generelle Frage, wie diese überhaupt sichergestellt werden könnte - z.B. über „ethnische“ oder migrantische Unternehmensverbände oder über eine Erhöhung der Sichtbarkeit von migrantischen Ökonomien in den entsprechenden Branchenverbänden.

Stadtentwicklungspolitik: Weder in Braunschweig noch in Rostock existiert eine erkennbare kommunalpolitische (Gesamt-) Strategie zur Förderung migrantischer Unternehmer*innen. Zwar gibt es in beiden Städten einzelne Aktivitäten, die genau diese Zielsetzung verfolgen (Räuchle, 2016). So dient in Rostock ein monatlich stattfindender „Existenzgründer- und Unternehmerstammtisch“ migrantischen Unternehmer*innen als Informationsplattform und bietet Zugang zu lokalen Entscheidungsträger*innen. Insgesamt werden migrantische Ökonomien jedoch selten direkt adressiert - und wenn, dann fast ausschließlich in Form von Beratungs- und Qualifizierungsangeboten (z.B. Coachings), 
die individuelle Unternehmer*innen (mit Migrationshintergrund), nicht jedoch die Gesamtheit der lokalen Rahmenbedingungen unternehmerischen Handelns betreffen (Kloosterman und Rath, 2011).

Als lokalpolitisches Thema bzw. Handlungsfeld sind migrantische Ökonomien in den beiden Fallstudienstädten insgesamt kaum etabliert. Überdies sind die wenigen Aktivitäten und Maßnahmen, die sich gezielt an migrantische Unternehmer*innen wenden, eher der Integrations- und Sozialals der Wirtschafts- und Stadtentwicklungspolitik zuzuordnen. Diese Ergebnisse zum institutionellen Kontext und zur Akteurskonstellation, innerhalb derer sich migrantische Ökonomien entfalten, sind mit Blick auf die kommunalpolitische Handhabung von Migrationsfragen im Allgemeinen und migrantischen Ökonomien im Besonderen durchaus aufschlussreich. Insofern können sie auch einen Beitrag zur (Selbst-) Reflexion kommunalpolitischer Praxen leisten. Zugleich leiden sie - aus einer postmigrantischen Perspektive betrachtet - jedoch unter derselben Unzulänglichkeit der zugrundeliegenden Begriffe wie die im vorangegangenen Abschnitt thematisierte statistische Analyse. So lässt es sich zwar nicht zuletzt mit Referenz auf das - ebenfalls nicht unumstrittene - Governance-Konzept rechtfertigen, einzelne Individuen zu Akteursgruppen zusammenzufassen und auf dieser Grundlage dann politische Prozesse und Fragen der politischen Repräsentation zu analysieren (Nuissl und Heinrichs, 2011). Das ändert aber nichts an den bereits benannten konzeptionellen Unzulänglichkeiten einer Aggregation ausgewählter Unternehmer*innen qua Nationalität (oder Migrationshintergrund) zum Konstrukt der migrantischen Ökonomien - ganz abgesehen von den differenzmarkierenden oder sogar ausgrenzenden Effekten einer solchen Begriffsbildung. Neuere Formen sozialer Grenzziehung sowie latente oder manifeste Rassismen, deren Thematisierung aus postmigrantischer Perspektive unerlässlich ist, bleiben auf diese Weise ausgeblendet.

Hinzu kommt das aus postmigrantischer Perspektive formulierte Petitum, den Zusammenhang von Migration und neoliberaler Stadtentwicklung kritisch im Auge zu behalten, das für die hier dargestellte Forschung von besonderer Relevanz ist. Es verlangt gleich in zwei Hinsichten nach einer Einordung und Reinterpretation der skizzierten Fallstudienergebnisse zur Governance migrantischer Ökonomien. Denn zum einen entstammen diese einer Forschungsaktivität, die - in ihrem Bestreben, Perspektiven und Problemstellungen der kommunalen Praxis aufzugreifen - selbst eine „,neoliberale“ Potentialorientierung reproduziert und dabei so ließe sich aus der Perspektive einer kritischen Erkenntnistheorie noch ergänzen - sogar in den beiden migrantisches Unternehmertum nur in begrenztem Umfang begünstigenden Governance-Kontexten noch Spuren dessen findet, was sie sucht: nämlich ein stadtentwicklungspolitisches Potential migrantischer Ökonomien. Zum anderen ist aber auch festzustellen, dass im ,empirischen Feld“ von Rostock und Braunschweig eine Orientierung der maßgeblichen stadtent- wicklungspolitischen Akteure auf dieses Potential kaum festzustellen ist. Von einer neoliberal motivierten, potentialorientierten Inwertsetzung migrantischer Ökonomien kann daher keine Rede sein. Weder in Braunschweig noch in Rostock hat sich bislang eine intermediäre, an den Schnittstellen von Kommunalverwaltung, Wirtschaft und Zivilgesellschaft angesiedelte Infrastruktur zur Förderung migrantischer Unternehmer*innen herausgebildet (wie es im Sinne eines ,typisch neoliberalen“ Governancearrangements eigentlich zu erwarten wäre) - weder top-down, noch bottomup. Nichtsdestotrotz werden selbstredend auch migrantische Unternehmer*innen in neoliberale politische Praxen wie etwa die des „Förderns und Forderns“ eingebunden; sie werden dabei aber nicht aufgrund ihres ethno-kulturellen Hintergrunds oder ihrer Staatsbürgerschaft adressiert, sondern allein aufgrund bestimmter zugeschriebener Kompetenzen bzw. Defizite: „Während Stadtpolitiken im Wohlfahrtsstaat die nationale Verteidigung der Gesellschaft betrieben (...), steht heute mit dem neoliberalen Funktionswandel von sozialem Ausgleich im Allgemeinen und mit der Aufwertung der Stadt als unternehmerische Einheit im internationalen Wettbewerb die nationale Form dieser Politiken zur Disposition und damit eröffnet der neoliberal turn selbst politische Spielräume für eine ,kosmopolitische "Entfaltung von citizenship“ (Rodatz, 2014: 46). Die Grenzen zwischen migrantisch und nicht-migrantisch werden damit aufgehoben. In diesem Sinne lässt sich das empirisch festgestellte Widerstreben vieler kommunaler Akteure gegen eine explizite Adressierung migrantischer Ökonomien dann doch als Ausdruck einer neoliberalen - und zugleich postmigrantischen - Stadtentwicklungspolitik verstehen.

\subsection{Sozio-kultureller Kontext: Deutungsmuster migrantischer Ökonomien}

Insbesondere mit Hilfe des Ansatzes der interpretativen Policy-Analyse wurde in den vergangenen Jahren überzeugend nachgewiesen, welchen großen Einfluss die Wahrnehmungs- und Einstellungsmuster lokaler Akteure darauf haben, wie kommunale Politik konkret gestaltet wird (Münch, 2016). Dies gilt auch für die lokalpolitische Handhabung migrantischer Ökonomien (Glick Schiller und Çağlar, 2013; Högberg et al., 2016). Vor diesem Hintergrund interessierte sich das hier diskutierte Forschungsprojekt auch dafür, wie migrantische Ökonomien von verschiedenen Akteuren - bzw. seitens der Akteursgruppen „Wirtschaft und Stadtentwicklung“, „Integration und Soziales“ sowie „migrantische Unternehmer*innen“ selbst - wahrgenommen werden. Dies wurde mittels einer Deutungsmusteranalyse untersucht, die Wahrnehmungen als soziales Phänomen versteht und ,quer“ zu einzelnen Interviewten herausarbeitet (Ullrich, 1999). Im Ergebnis konnten vier Deutungsmuster zur Rolle von migrantischen Ökonomien für die Stadtentwicklung identifiziert werden, die für Akteure in Braunschweig und Rostock handlungsleitend sind. 
Erstens werden migrantischen Ökonomien durchaus stadtentwicklungspolitische Potentiale attestiert. Hervorgehoben wird insbesondere, dass sie ,unternehmerische Vielfalt“ “ so der Ausdruck eines befragten IHK-Mitarbeiters - in eine Stadt bringen würden. Dies wirke sich positiv auf die Entwicklung der lokalen Wirtschaft aus. Vertreter*innen aller Akteursgruppen erkennen in migrant*innengeführten Geschäften und Dienstleistungen außerdem einen weichen Standortfaktor, da sie - wirtschaftlich - zur (besseren) Versorgung von Quartieren sowie - kulturell - zur (Belebung der) städtischen Atmosphäre beitrügen. Des Weiteren bescheinigen vor allem Befragte aus dem Bereich „Wirtschaft und Stadtentwicklung" migrantischen Ökonomien einen ausgeprägten Unternehmergeist sowie umfangreiche Unterstützungsnetzwerke aus Familienmitgliedern und Freund*innen. Die Wahrnehmung dieser Potentiale wird dabei häufig mit ethno-kulturellen Zuschreibungen verknüpft. Ähnliches wurde im Übrigen auch in anderen Studien beobachtet (z.B. Pütz, 2004). Vor allem migrantische Unternehmer*innen selbst heben darüber hinaus ihre besondere Kompetenz hervor, mit unterschiedlichen wirtschaftlichen, rechtlichen und sozialen Kontexten vertraut zu sein.

Zweitens werden die den migrantischen Ökonomien zugesprochenen Potentiale häufig wieder relativiert und infrage gestellt. Wiederum sind es insbesondere Akteure aus dem Bereich „Wirtschaft und Stadtentwicklung“, die unzureichende Bildung(sabschlüsse) und geringe Innovationsfähigkeit als unter einigen migrantischen Unternehmer*innen verbreitete Defizite kennzeichnen. Diese Wahrnehmung verbindet sich häufig mit einer spezifischen „Lesart“ migrantischer Ökonomien: Die unternehmerische Selbständigkeit von Migrant*innen wird als „Notlösung“ interpretiert, die auf fehlende Chancen auf dem Arbeitsmarkt zurückzuführen sei und in der Regel lediglich Kleinstbetriebe hervorbringe. Eine zweite, ebenfalls verbreitete Variante des „Notlösungsnarrativs“ interpretiert die unternehmerische Selbständigkeit von Migrant*innen nicht als Ausdruck individueller Defizite, sondern als Ergebnis einer Diskriminierung auf dem Arbeitsmarkt: Dann wird kritisiert, dass vielen Migrant*innen trotz eines hohen Bildungs- und Qualifikationsniveaus der Zugang zum ersten Arbeitsmarkt verwehrt werde.

Drittens prägt die Frage, ob migrantische Unternehmer*innen besonderer Unterstützung bedürfen, die Wahrnehmung migrantischer Ökonomien. Die beiden Fallstudien spiegeln damit eine der zentralen Schwerpunktsetzungen akademischer und politischer Debatten rund um migrantische Ökonomien wider (Rath und Swagerman, 2016). Dabei stehen die befragten Expert*innen einer ,Sonderförderung“ migrantischer Unternehmer*innen überwiegend kritisch gegenüber. Diese wird als (positiv) diskriminierend wahrgenommen, da Unternehmer*innen mit und ohne Migrationshintergrund in vielerlei Hinsicht ähnliche Schwierigkeiten bei der Gründung und Führung ihres Unternehmens zu gewärtigen hätten. Gleichwohl stimmen die meisten Expert*innen überein, dass migrantische Unternehmer*innen durchaus auch mit spezifischen Hindernissen konfrontiert seien, die beseitigt werden sollten. Nicht zuletzt sei es erforderlich, die hohen Hemmschwellen abzubauen, die migrantische Unternehmer*innen offenbar davon abhalten würden, sich an Behörden oder andere (halb-) öffentliche Institutionen wie Handels- und Handwerkskammern zu wenden, um sich beraten zu lassen.

Viertens hinterfragen die interviewten Akteure die Unterscheidung zwischen migrantischem und nichtmigrantischem Unternehmertum oder lehnen den Begriff der migrantischen Ökonomien sogar ausdrücklich ab. Dies wird mit zwei Argumenten begründet: Einige Interviewpartner*innen weisen auf die Heterogenität der unternehmerischen Aktivitäten von Migrant*innen sowie auf deren Nichtunterscheidbarkeit von nicht-migrantischen Wirtschaftsaktivitäten hin. Andere lehnen den Begriff der migrantischen Ökonomien aus normativen Gründen ab - sei es aufgrund der Überzeugung, dass für die Beurteilung von (erfolgreichem) Unternehmertum ausschließlich Kriterien wie Leistungsfähigkeit, Kreativität und Innovationskraft relevant seien, oder sei es aufgrund der grundsätzlich als diskriminierend wahrgenommenen Kennzeichnung von Menschen als Migrant*innen. Beide Argumente konvergieren in einem Deutungsmuster, das dem Konzept der migrantischen Ökonomien die Relevanz für die lokale Politikpraxis abspricht.

Im Rahmen der Analyse von Wahrnehmungs- und Einstellungsmustern lokaler Akteure wurde deutlich, dass Elemente eines ,postmigrantischen Paradigmas“ durchaus auch die kommunale Praxis prägen. Ein auf Fragen der Stadtentwicklung oder der kommunalen Wirtschaftsförderung bezogenes Deutungsmuster, in dem der Begriff der migrantischen Ökonomien eine wesentliche Rolle spielt, lässt sich dort nicht vorfinden. Stattdessen wird dieser Begriff von einer Reihe von Akteuren - teils aus normativen, teils aus inhaltlichen Gründen - zurückgewiesen. Allerdings dient das „Migrantische“ immer wieder auch als Referenzpunkt, um Differenzierungen vorzunehmen und lokalökonomische Sachverhalte zu beschreiben. So wird angesprochen, dass Migrant*innen häufig mit Diskriminierungen auf dem Arbeitsmarkt konfrontiert seien, und auch - nicht selten stereotype - Zuschreibungen zu migrantischen Unternehmer*innen sind in großer Zahl nachweisbar. Insofern wird exemplarisch deutlich, wie schwierig nicht zuletzt ein sprachlicher Umgang mit Migrationsphänomenen ist, der sowohl reflektiert und inklusiv als auch gegenstands- und praxisbezogen ist.

\section{Diskussion}

Als Ergebnis der Reflexion der Ansätze und Befunde des vorgestellten empirischen Forschungsprojekts lässt sich festhalten, dass diese einer postmigrantischen Kritik zumindest teilweise Stand halten. So konnte - im Sinne einer moderat postmigrantischen Perspektive, die von einer vorgängi- 
gen Definition dessen, was als migrantisch zu „labeln“ ist, absieht - herausgearbeitet werden, dass in der kommunalen Praxis etablierte Demarkationslinien zwischen migrantisch und nicht-migrantisch zwar teilweise reproduziert, vielfach aber auch infrage gestellt werden. Es bleibt aber grundsätzlich offen, inwiefern migrantische Ökonomien aus postmigrantischer Perspektive überhaupt ein sinnvolles Forschungsfeld konstituieren können.

Für die weitere wissenschaftliche Auseinandersetzung mit migrantischen Ökonomien spricht, dass sich in postmigrantischer Perspektive neue Fragen stellen, die zur kritischen Weiterentwicklung dieses Forschungsfelds beitragen. Erstens wird mit neuer Intensität zur Debatte über Begriffe und Definitionen aufgefordert, die sich eben jenseits von dichotomen Zuschreibungen bewegen. Zwar sind die Schwierigkeiten einer angemessenen begrifflichen Fassung der verschiedenen Aspekte zu migrantischen Ökonomien in der Forschung nicht neu und unter anderem als Othering oder Ethnisierung problematisiert worden. Mit der postmigrantischen Perspektive werden nun aber weitere Fragen nach sozialen Ungleichheiten, Hegemonie- und Dominanzstrukturen aufgeworfen. Das Konzept des Postmigrantischen wendet sich zweitens gegen eine Verengung des Integrationsbegriffs auf Migrant*innen und ihre Nachkommen (Foroutan, 2016). Übertragen auf das Feld migrantischer Ökonomien impliziert dies zum einen, dass nicht (mehr) primär danach zu fragen ist, wie die unternehmerische Tätigkeit von Migrant*innen zu deren Integration in unterschiedliche gesellschaftliche Teilsysteme beiträgt, sondern dass diese Tätigkeit in all denjenigen Facetten analysiert werden sollte, die auch für die Untersuchung der ökonomischen Aktivitäten von Nicht-Migrant*innen forschungsleitend sind. Zum anderen gilt es auch letztere daraufhin zu untersuchen, inwiefern sie migrantisch geprägt sind und/oder gesellschaftliche Integration (oder Desintegration) befördern. Im Sinne eines „strategischen Essentialismus“ (Mackenthun, 2017) erscheint es drittens - gerade in Zeiten zunehmender Xenophobie - wichtig, migrantische Unternehmer*innen und deren Leistungen sichtbar zu machen. Dieses einer postmigrantischen Herangehensweise latent widersprechende Anliegen lässt sich mit dem dezidiert postmigrantischen Erkenntnisinteresse am Beitrag alltäglicher transnationaler Praxen zur Produktion urbaner Räume verbinden (Yildiz, 2011). Viertens gilt es im Sinne der Forderung, Gesellschaftsforschung zu ,migrantisieren“, auch mit Blick auf lokale Ökonomien danach zu fragen, wie mit dem Verweis auf „Migration“ Grenzen gezogen, ,Andersartigkeiten“ begründet und Dominanzpositionen hergestellt und damit ethnisierende und kulturalisierende Handlungspraxen fortgeschrieben werden. Im Mittelpunkt des Interesses steht dann nicht die Gruppe der als migrantisch deklarierten Unternehmer*innen selbst, sondern die Frage, wie sich gesellschaftliche Akteure auf „Migration“ beziehen und damit ihr Handeln begründen. Hinzu kommen Fragen nach dem strategischen Nutzen und der Inszenierung von Ethnizität als Ressource im unternehmerischen Handeln (z.B. Stock, 2013).

Gegen die Erforschung migrantischer Ökonomien spricht erstens, dass es nicht möglich ist, der ihr inhärenten Markierung von sozialen Phänomenen oder Personen als ,anders“ zu entkommen. „Migrantisches“ in der Ökonomie wird damit eben nicht zur unhinterfragten gesellschaftlichen Normalität. Zudem ist es problematisch, wenn migrantische Ökonomien gleichsam essentialistisch als Kategorie, die einen ,realen“ Gegenstand bezeichnet, verstanden und der Analyse zugrunde gelegt werden. Zweitens und eng damit verknüpft ist zu hinterfragen, ob die Erforschung migrantischer Ökonomien nicht eine negative Konnotation von Migrationsphänomenen beibehält. So konstatiert Mecheril (2014: 109), dass eine instrumentelle Perspektive auf die Potentiale von Migration immer auch eine Unterscheidung zwischen ,guten“ und „schlechten“ Migrant*innen impliziere. Denn als Kehrseite der Betonung von Potentialen wird immer auch das Gegenteil - die Annahme scheinbarer Defizite - fortgeschrieben. Gerade in einem so kontroversen Feld wie dem der Migration kann Forschung politisch nicht neutral sein, sondern ist aufgefordert, stets zu reflektieren, inwiefern sie zur Verfestigung (oder zum Aufbrechen) bestimmter Perspektiven beiträgt (Fiedler et al., 2017; Nieswand und Drotbohm, 2014).

Das Konzept der Postmigration erhebt den Anspruch, nicht nur analytisch, sondern als ein politisch-normatives auch praktisch relevant zu sein. Insofern stellt sich die Frage, inwiefern migrantische Ökonomien im Kontext einer postmigrantischen Stadtentwicklungspolitik adressiert werden können. In der Gegenrichtung ist es durchaus legitim, auch aus der Perspektive der angewandten Geographie zu fragen, welchen Mehrwert die kritisch-postmigrantische Reflexion von Forschungsansätzen und -ergebnissen zu migrantischen Ökonomien im Hinblick auf die kommunalpolitische und planerische Raumproduktion haben kann. Foroutan (2015) vertritt die Auffassung, dass eine Gesellschaft dann als postmigrantisch bezeichnet werden kann, wenn ,Strukturen, Institutionen und politische Kulturen nachholend (also postmigrantisch) an die erkannte Migrationsrealität angepasst werden, was mehr Durchlässigkeit und soziale Aufstiege, aber auch Abwehrreaktionen und Verteilungskämpfe zur Folge hat". Übertragen auf das Feld der migrantischen Ökonomien würde dies bedeuten, dass kommunalpolitische Institutionen sich stärker als bisher auf Migrant*innen ausrichten, sich ihnen öffnen und mit ihnen vernetzen. Dies bedeutet nicht, die Repräsentanz von migrantischen Unternehmer*innen in einem multikulturalistischen Sinn zu stärken, sondern vielmehr die Diversität der Gesellschaft politisch und institutionell sichtbar zu machen (was nicht zuletzt die Kammern betrifft). Dabei geht es allerdings auch darum, jeglichen integrationspolitisch gerahmten Paternalismus hinter sich zu lassen und Integration als gesamtgesellschaftliche Aufgabe zu verstehen, die keiner Zielgruppen bedarf. Eine postmigrantische Stadtentwicklungspolitik muss aber auch stärker als bisher konfliktträchtige Auseinandersetzungen um städtische Ressour- 
cen aushalten und sich dabei an einer „Utopie der Gleichheit, die außerhalb der Herkunft verhandelt wird“" orientieren (Foroutan, 2016: 247).

\section{Fazit}

Im vorliegenden Aufsatz wurden Ansatz und Ergebnisse eines Forschungsprojekts zu migrantischen Ökonomien in einer postmigrantischen Perspektive zur Diskussion gestellt. Es wurde argumentiert, dass eine postmigrantisch informierte wissenschaftliche Auseinandersetzung mit migrantischen Ökonomien zwar ansatzweise, aber - aufgrund der dezidiert ,prä-postmigrantischen“ Definition des Forschungsgegenstands - nicht vollumfänglich möglich ist. Zudem wurde die Frage angerissen, inwiefern eine postmigrantische Perspektive auf migrantische Ökonomien auch für die stadtentwicklungspolitische Praxis hilfreich sein kann.

Insgesamt wurde erkennbar, dass die kritische Reflexion eines Forschungsprojekts zu den Potentialen migrantischer Ökonomien aus postmigrantischer Perspektive hilfreich ist, um dessen Befunde in die gegenwärtig in der Migrationsforschung geführten Debatten einzuordnen und dabei zugleich hinsichtlich seines affirmativen Gehalts zu hinterfragen. Darüber hinaus zeigte sich, dass nicht nur die wissenschaftliche Analyse migrantischer Ökonomien, sondern auch deren stadtentwicklungspolitische Handhabung von einer explizit postmigrantischen Herangehensweise noch ein gutes Stück entfernt ist. Hervorzuheben ist aber auch, dass in der empirischen Analyse des hier behandelten Forschungsprojekts für die kommunalpolitische Praxis (ebenso wie für die Forschung zu migrantischen Ökonomien selbst) durchaus auch postmigrantische Argumentationsfiguren festgestellt werden konnten.

Datenverfügbarkeit. Der dieser Studie zugrundeliegende Datensatz kann bei den Autor*innen erfragt werden. Da er sensible personenbezogene Daten enthält und zudem als Grundlage weiterer Projekte dient, wird er nicht öffentlich zugänglich gemacht.

Interessenkonflikt. Die Autor*innen erklären, dass kein Interessenkonflikt besteht.

Danksagung. Wir bedanken uns bei der Deutschen Forschungsgemeinschaft (DFG) für die Förderung des Projekts „Migrantische Ökonomie als Potential der Stadt- und Regionalentwicklung“, auf dem dieser Beitrag beruht. Des Weiteren bedanken wir uns bei den Organisatorinnen der Fachsitzung „Postmigrantische Stadt? Stadtgeographie und reflexiver Turn der Migrationsforschung“ auf dem DKG 2017 in Tübingen für die Möglichkeit der Präsentation unserer Ergebnisse sowie die Einladung zur Mitwirkung an dem vorliegenden Special Issue der GH. Schließlich geht unser Dank an zwei anonyme Reviewer*innen für ihre konstruktive Kritik und ihre hilfreichen Kommentare zu einer früheren Fassung des vorliegenden Beitrags.

Edited by: Karin Wiest

Reviewed by: two anonymous referees

\section{Literatur}

Aring, J. und Reuther, I.: Regiopolen: Die kleinen Großstädte in Zeiten der Globalisierung, Jovis-Verlag, Berlin, 2008.

Aver, C.: Migration, Ethnische Ökonomie und Stadtentwicklung, Informationen zur Raumentwicklung, 5, 393-402, 2013.

Aytar, V. und Rath, J. (Hrsg.): Selling Ethnic Neighborhoods, Routledge, London, New York, 2012.

Bertelsmann Stiftung: Migrantenunternehmen in Deutschland zwischen 2005 und 2014, Gütersloh, online aufrufbar: https://www. bertelsmann-stiftung.de/fileadmin/files/BSt/Publikationen/ GrauePublikationen/NW_Migrantenunternehmen.pdf (letzter Zugriff: 14 Dezember 2018), 2016.

Bojadzijev, M. und Römhild, R.: Was kommt nach dem „transnational turn"?, Perspektiven für eine kritische Migrationsforschung, in: Vom Rand ins Zentrum, Perspektiven einer kritischen Migrationsforschung, Berliner Blätter 65, Herausgeber: Labor Migration, Panama, Berlin, 10-24, 2014.

Dinnie, K. (Hrsg.): City Branding, Palgrave Macmillan, Houndmills Basingstoke, 2011.

Donald, S. H., Kofman, E., und Kevin, C. (Hrsg.): Branding Cities, Routledge, London, 2009.

Espahangizi, K., Hess, S., Karakayali, J., Kasparek, B., Pagano, S., Rodatz, M., und Tsianos, V. S.: Rassimus in der postmigrantischen Gesellschaft, Movements, 2, 9-23, 2016.

Fiedler, M., Georgi, F., Hielscher, L., Ratfisch, P., Riedner, L., Schwab, V., und Sontowski, S.: Umkämpfte Bewegungen nach und durch Europa, Movements, 3, 9-19, 2017.

Fischer-Krapohl, I.: Über die benachteiligten Stadtteile hinaus?, Raumsoziologie, 155, 73-77, 2011.

Fischer-Krapohl, I. und Waltz, V.: Migration als Chance der Städte, in: Stadtentwicklung mit Stadtmanagement? Herausgeber: Hochstadt, S., VS Verlag, Wiesbaden, 93-122, 2005.

Floeting, H.: Selbständigkeit von Migranten und informelle Netzwerke als Ressource für die Stadtentwicklung, in: Urban Recycling, Herausgeber: Yildiz, E. und Mattausch, B., Bauverlag, Gütersloh u.a., 52-62, 2009.

Foroutan, N.: Die postmigrantische Gesellschaft, online aufrufbar: http://www.bpb.de/gesellschaft/migration/kurzdossiers/ 205190/die-postmigrantische-gesellschaft (letzter Zugriff: 26 Juni 2018), 2015.

Foroutan, N.: Postmigrantische Gesellschaften, in: Einwanderungsgesellschaft Deutschland, Herausgeber: Brinkmann, H. U. und Sauer, M., Springer VS, Wiesbaden, 227-254, 2016.

Fürst, A. und Balke, J.: Transnationales ethnisches Unternehmertum, Raumforschung und Raumordnung, 71, 247-259, 2013.

Glasze, G. und Pott, A.: Räume der Migration und der Migrationsforschung, in: Räumliche Auswirkungen der Migration, Herausgeber: Gans, P., ARL, Hannover, 47-62, 2014.

Glick Schiller, N. und Çağlar, A. (Hrsg.): Locating Migration: Rescaling Cities and Migrants, Cornell University Press, Ithaca, 2011.

Glick Schiller, N. und Çağlar, A.: Locating Migrant Pathways of Economic Emplacement, Ethnicities, 13, 494-514, 2013. 
Harvey, D.: From Managerialism to Entrepreneurialism, Geogr. Ann. B, 71, 3-17, 1989.

Häußermann, H. und Kapphan, A.: Integrationspolitik der Städte, in: Migrationsreport 2008, Herausgeber: Bommes, M. und Krüger-Potratz, M., Campus, Frankfurt a.M., New York, 15-47, 2008.

Henn, S. und Behling, M. (Hrsg.): Aspekte integrierter Stadtteilentwicklung, Frank \& Timme, Berlin, 2010.

Hillmann, F.: Marginale Urbanität - Eine Einführung, in: Marginale Urbanität, Herausgeber: Dies., transcript, Bielefeld, 9-21, 2011.

Hillmann, F. und Sommer, E.: Döner und Bulette revisited, in: Marginale Urbanität, Herausgeber: Hillmann, F., transcript, Bielefeld, 23-86, 2011.

Högberg, L., Schölin, T., Ram, M., und Jones, T.: Categorising and Labelling Entrepreneurs, International Small Business Journal, 34, 242-260, 2016.

Husseini de Araújo, S. und Weber, F.: „Migrantenökonomien“ zwischen Wirtschaftsförderung und Diskriminierung, in: Räumliche Auswirkungen der internationalen Migration, Herausgeber: Gans, P., ARL, Hannover, 365-380, 2014.

Idik, E. und Schnetger, M.: Barrieren einer Migrantenökonomie und Bedingungen einer geeigneten Förderstruktur, in: Lokale Beschäftigung und Ökonomie, Herausgeber: Hanesch, W. und Krüger-Conrad, K., VS Verlag, Wiesbaden, 163-183, 2004.

Ipsen, D., Debik, J., Glasauer, H., Mussel, C., und Weichler, H.: Toronto, Arbeitsberichte Architektur, Stadtplanung, Landschaftsplanung Nr. 160, Universität Kassel, Kassel, 2005.

Kavaratzis, M. und Ashworth, G. J.: City Branding, TESG, 96, 506514, 2005

Kayser, P., Preusse, F., Riedel, J., und Umbreit, B.: Ethnische Ökonomie als Chance der Standortentwicklung, FHTW, Berlin, 2008.

Kloosterman, R. und Rath, J.: Immigrant Entrepreneurs in Advanced Economies: Mixed Embeddedness Further Explored, JEMS, 27, 189-201, 2011.

Leicht, R. und Langhauser, M.: Ökonomische Bedeutung und Leistungspotenziale von Migrantenunternehmen in Deutschland, Friedrich-Ebert-Stiftung, Bonn, online aufrufbar: http:// library.fes.de/pdf-files/wiso/10948.pdf (letzter Zugriff: 27 November 2017), 2014.

Light, I.: Ethnic Enterprise in America, University of California Press, Berkeley, Los Angeles, 1972.

Luk, C. M. und Phan, M. B.: Ethnic Enclave Reconfiguration, GeoJournal, 64, 17-30, 2005.

Mackenthun, G.: Essentialismus, strategischer, in: Handbuch Postkolonialismus und Literatur, Herausgeber: Göttsche, D., Dunker, A., und Dürbeck, G., J.B. Metzler Verlag, Stuttgart, 142-144, 2017.

Mecheril, P.: Was ist das X im Postmigrantischen?, sub \urban, 2, 107-112, 2014

Mestres, J.: Migrant Entrepreneurship in OECD Countries and its Contribution to Employment, in: Open for Business, Herausgeber: OECD, OECD Publishing, 23-62, https://doi.org/10.1787/9789264095830-en, 2010.

Münch, S.: Interpretative Policy-Analyse, Springer VS, Wiesbaden, 2016

Nieswand, B. und Drotbohm, H. (Hrsg.): Kultur, Gesellschaft, Migration, Springer VS, Wiesbaden, 2014.
Nuissl, H. und Heinrichs, D.: Fresh Wind or Hot Air?, J. Plan. Educ. Res., 31, 47-59, 2011.

Nuissl, H. und Schmiz, A.: Die migrantische Ökonomie als Potential der räumlichen Entwicklung, Geographische Rundschau, 67, 26-32, 2015.

Pécoud, A.: What is Ethnic in an Ethnic Economy?, Int. Rev. Mod. Sociol., 20, 59-76, 2010.

Portes, A., Haller, W., und Guarnizo, L. E.: Transnational Entrepreneurs, Am. Sociol. Rev., 67, 278-298, 2002.

Pütz, R.: Transkulturalität als Praxis, transcript, Bielefeld, 2004.

Puitz, R. und Rodatz, M.: Kommunale Integrations- und Vielfaltskonzepte im Neoliberalismus, Geogr. Z., 101, 166-183, 2013.

Ram, M., Trehan, K., Rouse, J., Woldesenbet, K., und Jones, T.: Ethnic Minority Business Support in the West Midlands, Environ. Plann. C, 30, 493-512, 2012.

Rath, J. und Eurofound: Promoting Ethnic Entrepreneurship in European Cities, Publications Office of the European Union, Luxembourg, 2011.

Rath, J. und Swagerman, A.: Promoting Ethnic Entrepreneurship in European Cities, Int. Migr., 54, 152-166, 2016.

Räuchle, C.: Migrantische Ökonomien in kleineren Großstädten, Berichte, 90, 135-156, 2016.

Rodatz, M.: Migration ist in dieser Stadt eine Tatsache, sub $\backslash$ urban, 2, 35-58, 2014.

Schaland, A.: Selbstständige Migrantinnen und Migranten in deutschen Städten, in: Urbanisierung und internationale Migration, Herausgeber: Kraas, F. und Bork, T., Nomos, Baden-Baden, 3344, 2012.

Schmitt, C.: Migrantisches Unternehmertum in Deutschland, transcript, Bielefeld, 2015.

Schmiz, A.: Transnationalität als Ressource?, transcript, Bielefeld, 2011.

Schmiz, A.: Staging a „Chinatown“ in Berlin, EURS, 24, 290-303, 2017.

Schuleri-Hartje, U.-.K., Floeting, H., und Reimann, B.: Ethnische Ökonomie, Schader-Stiftung und difu, Darmstadt, Berlin, 2005.

Shaw, S. J.: Marketing Ethnoscapes as Spaces of Consumption, Journal of Town \& City Management, 1, 381-395, 2011.

Stock, M.: Der Geschmack der Gentrifizierung: Arabische Imbisse in Berlin, transcript, Bielefeld, 2013.

Timm, E.: Kritik der „ethnischen Ökonomie“, PROKLA, 30, 363 376, 2000.

Ülker, B.: Enterprising Migrants in Berlin, transcript, Bielefeld, 2016.

Ullrich, C. G.: Deutungsmusteranalyse und diskursives Interview, Z. Soziol., 28, 429-447, 1999.

Unabhängige Kommission „Zuwanderung“: Zuwanderung gestalten - Integration fördern, Bericht der Unabhängigen Kommission „Zuwanderung“, Bundesministerium des Innern, Berlin, 2001.

Van der Horst, H. und Ouwehand, A.: „Multicultural Planning“ as a Contested Device in Urban Renewal and Housing, Urban Stud., 49, 861-875, 2012.

Waldinger, R. D., Aldrich, H. E., und Ward, R. (Hrsg.): Ethnic Entrepreneurs, Sage, Newbury Park, Kalifornien, 1990.

Yildiz, E.: Migration und Diversität als urbane Ressource, in: Die Besonderheit des Städtischen, Herausgeber: Herrmann, H., Keller, C., Neef, R., und Ruhne, R., VS Verlag, Wiesbaden, 125-143, 2011. 
Yildiz, E.: Postmigrantische Perspektiven, in: Pädagogisches Können in der Migrationsgesellschaft, Herausgeber: Doğmuş, A., Karakaşoğlu, Y., und Mecheril, P., Springer VS, Wiesbaden, 7184, 2016.

Yildiz, E. und Mattausch, B.: Urban Recycling, Bauwelt Fundamente 140, Bauverlag, Gütersloh u.a., 2009.
Zhuang, Z. C.: Ethnic Retailing and the Role of Municipal Planning, University of Waterloo, Ontario, PhD dissertation, 2008. 